\title{
Event-Triggered Adaptive Dynamic Programming Consensus Tracking Control for Discrete-Time Multiagent Systems
}

\author{
Yuyang Zhao, ${ }^{1}$ Xiaolin Dai, ${ }^{1}$ Dawei Gong, ${ }^{1}$ Xinzhi Lv, ${ }^{2}$ and Yang Liu $\mathbb{D}^{1}$ \\ ${ }^{1}$ School of Mechanical and Electrical Engineering, University of Electronic Science and Technology of China, \\ Chengdu 611731, China \\ ${ }^{2}$ Science and Technology on Reactor System Design Technology Laboratory, Nuclear Power Institute of China, \\ Chengdu 610213, China \\ Correspondence should be addressed to Yang Liu; ly2015@uestc.edu.cn
}

Received 17 December 2021; Revised 11 January 2022; Accepted 17 January 2022; Published 1 March 2022

Academic Editor: Chao Zeng

Copyright (c) 2022 Yuyang Zhao et al. This is an open access article distributed under the Creative Commons Attribution License, which permits unrestricted use, distribution, and reproduction in any medium, provided the original work is properly cited.

\begin{abstract}
This paper proposes a novel adaptive dynamic programming (ADP) approach to address the optimal consensus control problem for discrete-time multiagent systems (MASs). Compared with the traditional optimal control algorithms for MASs, the proposed algorithm is designed on the basis of the event-triggered scheme which can save the communication and computation resources. First, the consensus tracking problem is transferred into the input-state stable (ISS) problem. Based on this, the event-triggered condition for each agent is designed and the event-triggered ADP is presented. Second, neural networks are introduced to simplify the application of the proposed algorithm. Third, the stability analysis of the MASs under the event-triggered conditions is provided and the estimate errors of the neural networks' weights are also proved to be ultimately uniformly bounded. Finally, the simulation results demonstrate the effectiveness of the event-triggered ADP consensus control method.
\end{abstract}

\section{Introduction}

Because of the wide applications in the control field [1-6], the consensus control of MASs gained more and more attentions. In recent years, quite a few methods have been reported to solve the consensus control problem of MASs, such as adaptive control $[7,8]$ and sliding mode control $[9,10]$. It is worth mentioning that the previous methods focus on the stability of the MASs. However, the optimal characteristic is also worth considering in the consensus control problem. Optimal consensus control problem aims to find the optimal control policies which guarantee the stability of MASs and minimize the energy cost. As one of the core methods to achieve the optimal control policies, ADP approaches address the issue abovementioned by approximating the solutions of Hamilton-Jacobi-Bellman (HJB) equation [11-13].

Till now, ADP approaches have been applied in the optimal consensus control of MASs [14-20]. In [14], an optimal coordination control algorithm has been designed to address the consensus problem of the multiagent differential games through fuzzy ADP. The optimal output heterogeneous MASs was considered in [15]. Based on this work, Gao et al. [16] considered the dynamic uncertainties factor in the cooperative output regulation problems. Zhang et al. $[17,18]$ considered the optimal consensus tracking control for discrete-time/continuous-time MASs. In order to address the optimal consensus problem for unknown MASs with input delay, the authors proposed a data-driven disturbed adaptive controller based on ADP technique in [19]. In [20], the problem of data-based optimal consensus control was studied for MASs with multiple time delays. All the above results are based on the assumption that the communication and computing resources are big enough to transmit system data and update the control policy in every time step. However, it is difficult to be satisfied in practice.

Event-triggered control (ETC) is a well-recognized technology to address the above issue [21-24]. Different from the time-triggered control, whether the systems sample the signals or not only depends on the event-triggered 
condition. If it is satisfied at some time instants, then the data will be transmitted and the control policy will be updated. Therefore, compared with the control algorithms based on time-triggered scheme, the event-trigger control algorithms can efficiently save the computation resources [25]. In the past years, ETC is introduced to solve the optimal control problem under the limited computing resources [26-29]. In [26], an ETC method based on ADP is developed for continuous-time MASs. The authors considered the unknown internal states factor in the event-triggered optimal control for continuous-time MASs in [27]. The multiplayer zero-sum differential games are considered in [28] and an optimal consensus tracking control based on event-triggered is designed to solve this problem. In [29], an event-triggered optimal control algorithm is designed for unmatched uncertain nonlinear continuous-time systems. In [30], to save the limited network resources, an event-triggered mechanism was introduced to address the consensus problem of linear discrete-time MASs. The authors considered the event-triggered consensus problem of discrete-time multiagent networks in [31]. It is worthy to say, all the results in [26-29] studied the event-triggered optimal control for continuous-time MASs, but there were few works [30, 31] which consider the discrete-time MASs.

Motivated by the above discussions, an event-triggered ADP control algorithm is designed to address the optimal consensus tracking problem for discrete-time MASs. The major contributions of this paper are emphasized as follows:

(1) Comparing with the existing event-triggered ADP consensus control methods [27-29], we design the adaptive ET condition for every agent in the MASs. Then, the agent samples the data and communicates with the neighbors only when its event-triggered condition is satisfied. That means the agents in the MASs may not communicate with their neighbors or update their control policies at the same time instant, and then, the communicate resources are saved.

(2) In this paper, we give the stability analysis for the MASs under the event-triggered condition. It shows all agents in the discrete-time MASs will achieve consensus under the ET condition. And, we also prove the weight estimate errors for the critic neural networks (NNs) and actor NNs are uniformly ultimately bounded during the learning process.

The rest of this paper is organized as follows. In Section 2, the discrete-time MASs are considered and the consensus problem is formed. The event-triggered conditions for each agent in the system are introduced and the stability analysis is given in Section 3. Then, NN-based event-triggered ADP algorithm is introduced in Section 4, and the simulation results of this algorithm are given in Section 5. Finally, the conclusions are shown in Section 6.

\section{Problem Formation}

Consider the discrete-time MASs:

$$
\mathbf{x}_{i}(k+1)=\mathbf{A} \mathbf{x}_{i}(k)+\mathbf{B}_{i} \mathbf{u}_{i}(k),
$$

where $\mathbf{x}_{i}(k) \in R^{n \times 1}$ and $\mathbf{u}_{i}(k) \in R^{m_{i} \times 1}$ denote the state and the coordination control of agent $i, i \in 1,2, \ldots, N$, respectively. $\mathbf{A} \in R^{n \times n}$ and $\mathbf{B}_{i} \in R^{n \times m_{i}}$ are the constant matrices.

The leader's dynamics function is defined as

$$
\mathbf{x}_{0}(k+1)=\mathbf{A} \mathbf{x}_{0}(k)
$$

where $x_{0}(k) \in R^{n}$ denotes the state of the leader.

The local neighbor consensus tracking error $\xi_{i}$ is defined as

$$
\xi_{i}(k)=\sum_{j \in \mathcal{N}_{i}} \alpha_{i j}\left(\mathbf{x}_{i}(k)-\mathbf{x}_{j}(k)\right)+\beta_{i}\left(\mathbf{x}_{i}(k)-\mathbf{x}_{0}(k)\right) .
$$

where $\alpha_{i j}$ denotes the adjacency elements, $a_{i j}>0$ if agent $i$ can communicate with agent $j$, otherwise, $\alpha_{i j}=0$, and $\beta_{i}$ denotes the pinning gain, $\beta_{i}>0$, if agent $i$ can communicate with the leader, otherwise, $\beta_{i}=0$. We assume that there is at least one agent who can get the information from the leader.

Under the event-triggered scheme, the discrete-time MASs transmit the systems' data only when the event is triggered. Here, we define that the event is triggered at the discrete-time instants' sequence $k_{i, 1}, k_{i, 2}, \ldots, k_{i, p-1}, k_{i, p}$, for $i=1,2, \ldots, N$ and $p=1,2, \ldots, \infty$. At the pth event-triggered instant of agent $i$, the consensus errors of agent $i$ denote as $\widehat{\xi}_{i}\left(k_{i, p}\right)=\sum_{j \in \mathcal{N}_{i}} \alpha_{i j}\left(\mathbf{x}_{i}\left(k_{\mathrm{i}, p}\right)-\mathbf{x}_{j}\left(k_{j, q}\right)\right)+\beta_{i}\left(\mathbf{x}_{i}\left(k_{i, p}\right)\right.$
$\left.-\mathbf{x}_{0}\left(k_{i, p}\right)\right)$.

The event-triggered error is defined as

$$
\boldsymbol{\delta}(k)=\widehat{\boldsymbol{\xi}}_{i}\left(k_{i, p}\right)-\boldsymbol{\xi}_{i}(k),
$$

which means the difference between the consensus tracking errors at the pth event-triggered instant and the current local neighbor consensus tracking errors.

Then, the consensus problem of the discrete-time MASs is to find the distributed feedback control law, $\mathbf{u}_{i}(k)=\chi\left(\widehat{\xi}_{i}\left(k_{i, p}\right)\right)$, which becomes a continuous signal through a zero-order hold (ZOH) device when $k_{i, p} \leq k<k_{i, p+1}$.

Then, the local cost function is defined as

$$
\begin{aligned}
J_{i}\left(\xi_{i}(k), u_{i}(k), u_{j}(k)\right) & =\sum_{l=k}^{\infty} \rho^{t-k} U_{i}\left(\xi_{i}(t), u_{i}(t), u_{j}(t)\right) \\
& =U_{i}\left(\xi_{i}(k), u_{i}(k), u_{j}(k)\right)+\rho J_{i}\left(\xi_{i}(k+1), u_{i}(k+1), u_{j}(k+1)\right),
\end{aligned}
$$

where 
(i) $U_{i}\left(\xi_{i}(k), \mathbf{u}_{i}(k), u_{j}(k)\right)$ : the utility function, for agent $i$,

$$
\begin{aligned}
U_{i}\left(\xi_{i}(k), \mathbf{u}_{i}(k), \mathbf{u}_{j}(k)\right)= & \xi_{i}^{\mathrm{T}}(k) \mathbf{Q}_{i i} \xi_{i}(k)+\mathbf{u}_{i}^{\mathrm{T}}(k) \mathbf{R}_{i i} \mathbf{u}_{i}(k) \\
& +\sum_{j \in \mathcal{N}_{i}} \mathbf{u}_{j}^{\mathrm{T}}(k) \mathbf{S}_{i j} \mathbf{u}_{j}(k) .
\end{aligned}
$$

(ii) $\mathbf{u}_{j}(k)$ : the control of the neighbors of agent $i$.

(iii) $\mathbf{Q}_{i i}, \mathbf{R}_{i i}$, and $\mathbf{S}_{i j}$ : positive symmetric weighting matrices.

(iv) $p$ : the discount factor, $0<\rho \leq 1$.
According to Bellman's principle, the optimal local cost function $J_{i}^{*}\left(\xi_{i}(k), \mathbf{u}_{i}(k), \mathbf{u}_{j}(k)\right)$ can be defined as

$$
\begin{aligned}
J_{i}^{*}\left(\xi_{i}(k), \mathbf{u}_{i}(k), \mathbf{u}_{j}(k)\right)= & \min _{u_{i}\left(\xi_{i}\left(k_{i, p}\right)\right)}\left\{U_{i}\left(\xi_{i}(k), \mathbf{u}_{i}(k), \mathbf{u}_{j}(k)\right)\right. \\
& \left.+\rho J_{i}^{*}\left(\xi_{i}(k+1), \mathbf{u}_{i}(k+1), \mathbf{u}_{j}(k+1)\right)\right\},
\end{aligned}
$$

which is also called discrete-time HJB equations.

The optimal disturbed control law $\mathbf{u}_{i}^{*}\left(\xi_{i}\left(k_{i, p}\right)\right)$ is defined as

$$
\mathbf{u}_{i}^{*}\left(\xi_{i}\left(k_{i, p}\right)\right)=\arg \min _{u_{i}\left(\xi_{i}\left(k_{i, p}\right)\right)}\left\{U_{i}\left(\xi_{i}(k), \mathbf{u}_{i}(k), \mathbf{u}_{j}(k)\right)+\rho J_{i}^{*}\left(\boldsymbol{\xi}_{i}(k+1), \mathbf{u}_{i}(k+1), \mathbf{u}_{j}(k+1)\right)\right) .
$$

\section{Stability Analysis}

Assumption 1 (see [32]). There exist positive constants $L, L_{1}$, $\phi$, and $\psi$, a $C^{1}$ function $V: R^{n} \longrightarrow R \geq 0$, and class $\kappa_{\infty}$ functions $\gamma_{1}$ and $\gamma_{2}$, such that

$$
\begin{gathered}
\|\mathbf{A}\| \leq L,\|\mathbf{B}\| \leq L, \| \chi\left(\boldsymbol{\xi}_{i}(k)+\boldsymbol{\delta}((k))\|\leq L\| \bar{\xi}_{i}\left(k_{i, p}\right) \|,\right. \\
\qquad \gamma_{1}(\|\mathbf{x}\|) \leq V(\mathbf{x}(k)) \leq \gamma_{2}(\|\mathbf{x}\|) \forall \mathbf{x} \in \mathbb{R}^{n}, \\
V\left(\mathbf{A} x_{i}(k)+\mathbf{B}_{i} \chi\left(\boldsymbol{\xi}_{i}(k)+\boldsymbol{\delta}(k)\right)\right)-V\left(\mathbf{x}_{i}(k)\right) \leq-\phi V\left(\mathbf{x}_{i}(k)\right)+\psi\|\boldsymbol{\delta}(k)\| . \\
\|\boldsymbol{\delta}(k+1)\| \leq\left\|\hat{\xi}_{i}\left(k_{i, p}\right)\right\|+\left\|\xi_{i}(k+1)\right\| .
\end{gathered}
$$

If (10) and (11) are satisfied, function $V$ is called an ISSLyapunov function for the discrete-time MAS.

Let us consider a situation that $k \in\left[k_{i, p}, k_{i, p+1}\right)$, which means that the ET condition is satisfied at the sampling instant $k_{i, p}$. In this situation, it is obvious that $\delta_{i, p}(k+1) \stackrel{i, p}{=} \widehat{\xi}_{i}\left(k_{i, p}\right)-\xi_{i}(k+1)$. Then, we have
Substituting (1) and (2) into (3), we have

$$
\begin{aligned}
\xi_{i}(k+1) & =\sum_{j \in \mathscr{N}_{i}} \alpha_{i j}\left(\mathbf{A}\left(\mathbf{x}_{i}(k)-\mathbf{x}_{j}(k)\right)+\mathbf{B}_{i} \mathbf{u}_{i}(k)-\mathbf{B}_{j} \mathbf{u}_{j}(k)\right)+\beta_{i}\left(\mathbf{A}\left(\mathbf{x}_{i}(k)-\mathbf{x}_{0}(k)\right)+\mathbf{B}_{i} \mathbf{u}_{i}(k)\right) \\
& \left.=\mathbf{A} \xi_{i}(k)+\sum_{j \in \mathscr{N}_{i}} \alpha_{i j}\left(\mathbf{B}_{i} \mathbf{u}_{i}(k)-\mathbf{B}_{j} \mathbf{u}_{j}(k)\right)+\beta_{i} \mathbf{B}_{i} \mathbf{u}_{i}(k)\right) .
\end{aligned}
$$

Then, we can have 


$$
\begin{aligned}
\left\|\boldsymbol{\xi}_{i}(k+1)\right\| & \leq\|\mathbf{A}\|\left\|\boldsymbol{\xi}_{i}(k)\right\|+\sum_{j \in \mathcal{N}_{i}} \alpha_{i j}\left\|\left(\mathbf{B}_{i} \mathbf{u}_{i}(k)-\mathbf{B}_{j} \mathbf{u}_{j}(k)\right)\right\|+\beta_{i}\left\|\mathbf{B}_{i} \mathbf{u}_{i}(k)\right\| \\
& \leq\|\mathbf{A}\|\left\|\boldsymbol{\xi}_{i}(k)\right\|+\sum_{j \in \mathcal{N}_{i}} \alpha_{i j}\left\|\left(\mathbf{B}_{i} \mathbf{u}_{i}(k)-\mathbf{B}_{j} \mathbf{u}_{j}(k)\right)\right\|+\beta_{i}\left\|\mathbf{B}_{i} \mathbf{u}_{i}(k)\right\| \\
& \leq\|\mathbf{A}\|\left\|\boldsymbol{\xi}_{i}(k)\right\|+\sum_{j \in \mathcal{N}_{i}} \alpha_{i j}\left(\left\|\mathbf{B}_{i} \mathbf{u}_{i}(k)\right\|+\left\|\mathbf{B}_{j} \mathbf{u}_{j}(k)\right\|\right)+\beta_{i}\left\|\mathbf{B}_{i} \mathbf{u}_{i}(k)\right\| .
\end{aligned}
$$

Substituting (9) into (14), we have

$$
\begin{aligned}
\left\|\boldsymbol{\xi}_{i}(k+1)\right\| & \leq\|\mathbf{A}\|\left\|\boldsymbol{\xi}_{i}(k)\right\|+\sum_{j \in \mathcal{N}_{i}} \alpha_{i j}\left(\left\|\mathbf{B}_{i} \mathbf{u}_{i}(k)\right\|+\left\|\mathbf{B}_{j} \mathbf{u}_{j}(k)\right\|\right)+\beta_{i} L\left\|\mathbf{u}_{i}(k)\right\| \\
& \leq L\left\|\boldsymbol{\xi}_{i}(k)\right\|+L \sum_{j \in \mathcal{N}_{i}} \alpha_{i j}\left(\left\|\mathbf{u}_{j}(k)\right\|+\left\|\mathbf{u}_{i}(k)\right\|\right)+\beta_{i} L\left\|\mathbf{u}_{i}(k)\right\| \\
& \leq L\left\|\boldsymbol{\xi}_{i}(k)\right\|+L \sum_{j \in \mathcal{N}_{i}} \alpha_{i j}\left(\left\|\mathbf{u}_{j}(k)\right\|+L\left\|\hat{\xi}_{i}\left(k_{i, p}\right)\right\|\right)+\beta_{i} L^{2}\left\|\hat{\xi}_{i}\left(k_{i, p}\right)\right\| .
\end{aligned}
$$

Therefore,

$$
\begin{aligned}
& \|\boldsymbol{\delta}(k)\| \leq\left\|\hat{\xi}_{i}\left(k_{i, p}\right)\right\|+\left\|\boldsymbol{\xi}_{i}(k-1)\right\| \leq\left\|\hat{\xi}_{i}\left(k_{i, p}\right)\right\|+L\left\|\boldsymbol{\xi}_{i}(k-1)\right\| \\
& +L \sum_{j \in \mathscr{N}_{i}} \alpha_{i j}\left(\left\|\mathbf{u}_{j}(k)\right\|+L\left\|\hat{\xi}_{i}\left(k_{i, p}\right)\right\|\right)+\beta_{i} L^{2}\left\|\hat{\xi}_{i}\left(k_{i, p}\right)\right\| \\
& =\left\|\hat{\xi}_{i}\left(k_{i, p}\right)\right\|+\beta_{i} L^{2}\left\|\hat{\xi}_{i}\left(k_{i, p}\right)\right\|+L \sum_{j \in N_{i}} \alpha_{i j}\left(\left\|\mathbf{u}_{j}(k)\right\|+L\left\|\hat{\xi}_{i}\left(k_{i, p}\right)\right\|\right) \\
& +L\left\|\xi_{i}(k-1)\right\| \leq\left(1+\beta_{i} L^{2}\right)\left\|\hat{\xi}_{i}\left(k_{i, p}\right)\right\|+L \sum_{j \in \mathcal{N}_{i}} \alpha_{i j}\left(\left\|\mathbf{u}_{j}(k)\right\|+L\left\|\hat{\xi}_{i}\left(k_{i, p}\right)\right\|\right) \\
& +L\left(L\left\|\xi_{i}(k-2)\right\|+L \sum_{j \in \mathcal{N}_{i}} \alpha_{i j}\left(\left\|\mathbf{u}_{j}(k)\right\|+L\left\|\widehat{\xi}_{i}\left(k_{i, p}\right)\right\|\right)+\beta_{i} L^{2}\left\|\widehat{\xi}_{i}\left(k_{i, p}\right)\right\|\right) \\
& \cdots \leq\left(1+\beta_{i} L^{2}+\beta_{i} L^{3}+\cdots+\beta_{i} L^{k-k_{i, p}+1}\right)\left\|\hat{\xi}_{i}\left(k_{i, p}\right)\right\| \\
& +\left(1+L+\cdots+L^{k-k_{i, p}-1}\right) L \sum_{j \in \mathcal{N}_{i}} \alpha_{i j}\left(\left\|\mathbf{u}_{j}(k)\right\|+L\left\|\hat{\xi}_{i}\left(k_{i, p}\right)\right\|\right)+L^{k-k_{i, p}}\left\|\hat{\xi}_{i}\left(k_{i, p}\right)\right\| .
\end{aligned}
$$

Then, we can rewrite the ET condition as

$$
\begin{aligned}
\left\|\boldsymbol{\delta}_{i, p}(k)\right\| \leq & \delta_{i T}=\left(1+L^{k-k_{i, p}}\right)\left\|\tilde{\xi}\left(k_{i, p}\right)\right\|+\beta_{i} \frac{L^{2}\left(1-L^{k-k_{i, p}}\right)}{1-L}\left\|\tilde{\xi}\left(k_{i, p}\right)\right\| \\
& +L \frac{1-L^{k-k_{i, p}}}{1-L} \sum_{j \in N_{i}} \alpha_{i j}\left(\left\|\mathbf{u}_{j}(k)\right\|+L\left\|\hat{\xi}_{i}\left(k_{i, p}\right)\right\|\right),
\end{aligned}
$$

for every $k \in\left[k_{i, p}, k_{i, p+1}\right)$. 
To better illustrate the control process, a flowchart has been displayed in Figure 1. The transmitted data and control policies are updated at $k_{i, p}$ instant, and the event-triggered error is reset to zero. Once the event-triggered condition is satisfied, the current instant becomes the next triggering instant $k_{i, p+1}$, and the system data are transmitted.
Otherwise, keep the transmitted data and control policies unchanged.

Then, we will prove the discrete-time MAS is stable under our event-triggered conditions.

Theorem 1. If a discrete-time MASs which is under assumption 1 and satisfies the function,

$$
V(\mathbf{x}(k)) \leq V\left(\mathbf{x}\left(k_{i, p+1}\right)\right)=-\varphi \phi V\left(\left(\mathbf{x}\left(k_{i, p}\right)\right)\left(k_{i, p+1}-k_{i, p}\right)+\psi V\left(\mathbf{x}\left(k_{i, p}\right)\right),\right.
$$

for every $k \in\left[k_{i, p}, k_{i, p+1}\right)$, where $\varphi \in(0,1)$, then the system is

$$
V\left(\mathbf{A x}_{i}(k)+\mathbf{B}_{i} \chi\left(\xi_{i}(k)+\boldsymbol{\delta}(k)\right)\right) \leq(1-\phi) V\left(\mathbf{x}_{i}(k)\right)+\psi\|\boldsymbol{\delta}(k)\| .
$$

asymptotically stable.

Then, applying (18) into (19), we have

Proof. According to (9) and (11), we obtain

$$
\begin{aligned}
V\left(\mathbf{A x}_{i}(k)+\mathbf{B}_{i} \chi\left(\xi_{i}(k)+\boldsymbol{\delta}(k)\right)\right) \leq & (1-\phi) V\left(\mathbf{x}_{i}(k)\right)+\psi \boldsymbol{\delta}(k) \\
\leq & (1-\phi) V\left(\mathbf{x}_{i}(k)\right)+\psi\left(\left(1+L^{k-k_{i, p}}+\beta_{i} \frac{L^{2}\left(1-L^{k-k_{i, p}}\right)}{1-L}\right)\left\|\hat{\xi}_{i}\left(k_{i, p}\right)\right\|\right. \\
& \left.+L \frac{1-L^{k-k_{i, p}}}{1-L} \sum_{j \in \mathcal{N}_{i}} \alpha_{i j}\left(\left\|\mathbf{u}_{j}(k)\right\|+L\left\|\hat{\xi}_{i}\left(k_{i, p}\right)\right\|\right)\right) .
\end{aligned}
$$

Solving (20), we can obtain

$$
\begin{aligned}
V\left(\mathbf{A x}_{i}(k)+\mathbf{B}_{i} \chi\left(\boldsymbol{\xi}_{i}(k)+\boldsymbol{\delta}(k)\right)\right) \leq & (1-\phi)^{k-k_{i, p}} V\left(\mathbf{x}_{i}\left(k_{i, p}\right)\right) \\
+ & \psi \frac{1-(1-\phi)^{k-k_{i, p}}}{\phi}\left(\left(1+L^{k-k_{i, p}}+\beta_{i} \frac{L^{2}\left(1-L^{k-k_{i, p}}\right)}{1-L}\right)\left\|\widehat{\xi}_{i}\left(k_{i, p}\right)\right\|\right. \\
& \left.+L \frac{1-L^{k-k_{i, p}}}{1-L} \sum_{j \in \mathcal{N}_{i}} \alpha_{i j}\left(\left\|\mathbf{u}_{j}(k)\right\|+L\left\|\widehat{\xi}_{i}\left(k_{i, p}\right)\right\|\right)\right) .
\end{aligned}
$$

We define a function as

$$
F(\mathbf{x}(k))=-\varphi \phi V\left(\left(\mathbf{x}\left(\left(k_{i, p}\right)\right)\left(k-k_{i, p}\right)+\psi V\left(\mathbf{x}\left(k_{i, p}\right)\right), \quad \forall k \in\left[k_{i, p}, k_{i, p+1}\right) .\right.\right.
$$

According to (18), we have

$$
V(\mathbf{x}(k)) \leq F(\mathbf{x}(k)),
$$

for every $k \in\left[k_{i, p}, k_{i, p+1}\right)$.

From (22), we obtain

$\Delta F=F\left(\mathbf{x}(k+1)-F(\mathbf{x}(k))=-\varphi \phi V\left(\mathbf{x}\left(k_{i, p}\right)\right)\right)$.
Applying (9) into (24), we have

$$
\Delta F \leq-\varphi \phi \gamma_{1}\left\|\mathbf{x}\left(k_{i, p}\right)\right\| \forall k \in\left[k_{i, p}, k_{i, p+1}\right) .
$$

Since (23) and (25) hold, the stability of the discrete-time MAS is proved. 


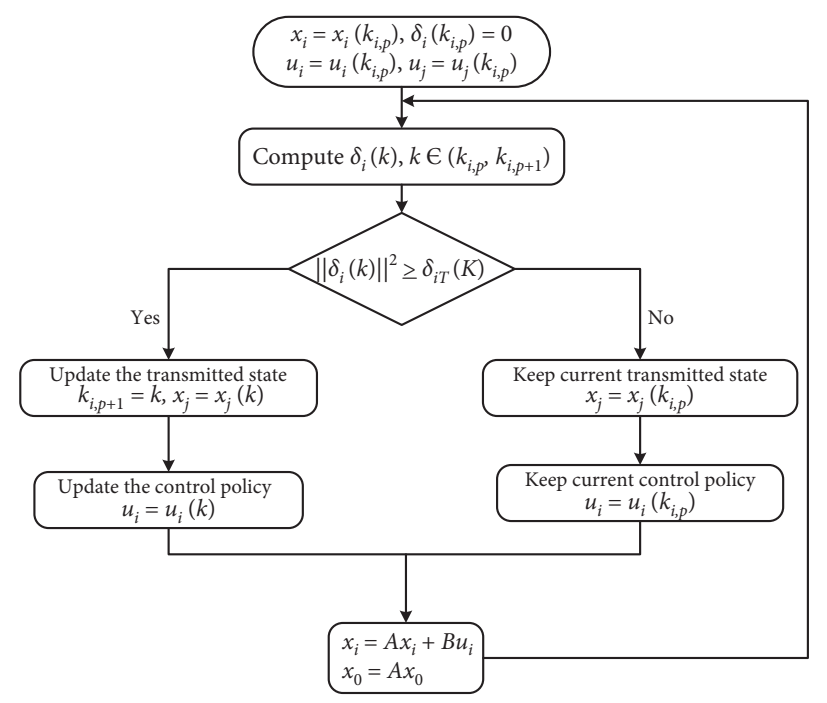

Figure 1: The flowchart of the event-triggered consensus control algorithm.

Remark 1. We give the event-triggered condition for each agent in the discrete-time MASs. Moreover, the stability of the systems is also proved in this paper.

\section{Event-Trggered Controller Design}

In this section, considering the good fitting characteristics of the neural networks (NN) [33,34], the actor-critic neural network structure is introduced to approximate the local cost function $J_{i}\left(\xi_{i}(k), u_{i}(k), u_{j}(k)\right)$ and the distributed feedback control law $u_{i}(x)$. The actor-critic NNs are defined as

$$
\widehat{\mathscr{F}}(\omega, z, w)=\omega^{\mathrm{T}} \Psi\left(w^{\mathrm{T}} z\right)=\omega^{\mathrm{T}} \Psi(Z),
$$

where $z$ denotes the input data, $\Psi(\cdot)$ denotes the activation functions, and $w$ and $\omega$ denote the weight matrices of the NNs.
4.1. Formulation of the Critic Networks. The critic NN approximates the local cost function $J_{i}\left(\xi_{i}(k), u_{i}(k), u_{j}(k)\right)$ in this paper as follows:

$$
\widehat{V}_{i}(k)=\widehat{\omega}_{c i}^{\mathrm{T}} \Psi_{c i}\left(\widehat{w}_{c i}^{\mathrm{T}} z_{c i}(k)\right),
$$

where $z_{c i}(k)$ denotes the input vector of the critic NN which is constituted by $\xi_{i}(k), u_{i}(k)$, and $u_{\mathcal{N}(i)}(k), \Psi_{c i}(\cdot)$ denotes the activation function of the critic NN, and $\widehat{w}_{c i}$ and $\widehat{\omega}_{c i}$ are the weight matrices for the critic NN.

We define the difference between the current cost value and the estimate value as the error function of the critic NN as follows:

$$
\varepsilon_{c i}(k)=-U_{i}\left(\xi_{i}(k), u_{i}(k), u_{j}(k)+\rho \widehat{\omega}_{c i}^{T} \Psi_{c i}\left(\widehat{w}_{c i}^{T} z_{c i}(k+1)\right)\right)+\widehat{\omega}_{c i}^{T} \Psi_{c i}\left(\widehat{w}_{c i}^{T} z_{c i}(k)\right) .
$$

Then, the loss function for the critic NN is given as

$$
E_{c i}=\frac{1}{2} \varepsilon_{c i}^{\mathrm{T}}(k) \varepsilon_{c i}(k)
$$

Our objective is to minimize the loss function during the critic NN training.

The weights for the critic NN are updated according to the gradient-based rule, which is given as follows:

$$
\begin{aligned}
\widehat{\omega}_{c i}(k+1)= & \widehat{\omega}_{c i}(k)-\mathscr{K}_{c i} \frac{\partial E_{c i}(k)}{\partial \varepsilon_{c i}(k)} \frac{\partial \varepsilon_{c i}(k)}{\partial \widehat{\omega}_{c i}(k)} \\
= & \widehat{\omega}_{c i}(k)-\mathscr{K}_{c i} \varepsilon_{c i}^{T}(k)-\rho \psi_{c i}\left(\widehat{w}_{c i}^{T} z_{c i}(k+1)\right) \\
& +\psi_{c i}\left(\widehat{w}_{c i}^{T} z_{c i}(k)\right),
\end{aligned}
$$

where $\mathscr{K}_{c i}$ denotes the learning rate.

4.2. Formulation of the Actor Networks. The actor NN approximates the disturbed control law $u_{i}(k)$, which can be formulated as

$$
\widehat{u}_{i}(k)=\widehat{\omega}_{a i}^{T} \Psi_{a i}\left(\widehat{w}_{a i}^{T} z_{a i}(k)\right),
$$

where $z_{a i}(k)$ is the input vector of the actor NN, $\psi_{a i}(\cdot)$ is the activation function for the actor $\mathrm{NN}$, and $\widehat{\omega}_{a i}$ and $\widehat{w}_{a i}$ are the weight matrices for the actor NN.

We define the difference between the current local cost value $\widehat{V}_{i}(k)$ and the target cost value $P_{i}(k)$ as the error function, which is given as

$$
\varepsilon_{a i}=\widehat{V}_{i}(k)-P_{i}(k) .
$$


In this paper, the target cost value is defined as 0 .

Then, the loss function for the actor $\mathrm{NN}$ is given as

$$
E_{a i}=\frac{1}{2} \varepsilon_{a i}^{T}(k) \varepsilon_{a i}(k) .
$$

Our objective is to minimize the loss function during the actor NN training.

The weights for the actor $\mathrm{NN}$ is updated according to the gradient-based rule, which is given as follows:

$$
\begin{aligned}
\widehat{\omega}_{a i}(k+1) & =\widehat{\omega}_{a i}(k)-\mathscr{K}_{a i} \frac{\partial E_{a i}(k)}{\partial \varepsilon_{a i}(k)} \frac{\partial \varepsilon_{a i}(k)}{\partial \widehat{V}_{i}(k)} \frac{\partial \widehat{V}_{i}(k)}{\partial \widehat{u}_{i}(k)} \frac{\partial \widehat{u}_{i}(k)}{\partial \widehat{\omega}_{a i}(k)} \\
& =\widehat{\omega}_{a i}(k)-\mathscr{K}_{a i} \varepsilon_{a i}^{T}(k) \widehat{\omega}_{c i} \Omega(k) C_{i} \Psi_{a i}\left(\widehat{\omega}_{a i}^{T} z_{a i}(k)\right),
\end{aligned}
$$

where $\Omega(k)=\partial \Psi_{c i}\left(\widehat{w}^{T}{ }_{c i} z_{c i}\right) / \partial z_{c i}, C_{i}=\partial z_{c i} / \partial u_{i}$, and $\mathscr{K}_{a i}$ is the learning rate for the actor $\mathrm{NN}$.

The procedure of the NN-based event-triggered optimal consensus control algorithm for discrete-time MASs is shown in Algorithm 1.

Theorem 2. Consider a discrete-time MAS. The weights of critic NN and actor NN are updated following (30) and (34), respectively, under condition (17). The state $x_{i}$, the critic NN weight estimation error, $\widetilde{\omega}_{c i}=\omega_{c i}-\omega_{c i}^{*}$, and the action weight estimation error, $\widetilde{\omega}_{a i}=\omega_{a i}-\omega_{a i}^{*}$, in the close loop system are UUB.

Proof

Case 1: the ET condition is satisfied at iteration index $k$. The Lyapunov function for agent $i$ can be defined as follows:

$$
\mathscr{L}_{i}(k)=\mathscr{L}_{i, 1}(k)+\mathscr{L}_{i, 2}(k)+\mathscr{L}_{i, 3}(k),
$$

where $\quad L_{i, 1}(k)=\mathbf{x}_{i}^{T}(k) \mathbf{x}_{i}(k), \quad L_{i, 2}(k)=\left(1 / \mathscr{K}_{c i}\right) t r$ $\left\{\widetilde{\omega}_{c i}^{T}(k) \widetilde{\omega}_{c i}(k)\right\}$, and $L_{i, 3}(k)=1 / \mathscr{K}_{a i} \cdot \operatorname{tr}\left\{\widetilde{\omega}_{a i}^{T}(k) \widetilde{\omega}_{a i}(k)\right\}$. The difference between $\mathscr{L}_{i, 1}(k+1)$ and $\mathscr{L}_{i, 1}(k)$ can be given as

$$
\begin{aligned}
\Delta \mathscr{L}_{i, 1} & =\mathbf{x}_{i}^{T}(k+1) \mathbf{x}_{i}(k+1)-\mathbf{x}_{i}^{T}(k) \mathbf{x}_{i}(k), \\
& =\left\|\mathbf{x}_{i}(k+1)\right\|^{2}-\left\|\mathbf{x}_{i}(k)\right\|^{2}, \\
& =-\left\|\mathbf{x}_{i}(k)\right\|^{2}+\left\|\mathbf{A}_{i} \mathbf{x}_{i}(k)+\mathbf{B}_{i} \omega_{a i}^{*} \Psi\left(w_{a i} \xi_{i}\right)\right\|^{2} .
\end{aligned}
$$

The difference between $\mathscr{L}_{i, 2}(k+1)$ and $\mathscr{L}_{i, 2}(k)$ can be given as

$$
\begin{aligned}
\Delta \mathscr{L}_{i, 2} & =\left(1 / \mathscr{K}_{c i}\right) \operatorname{tr}\left\{\widetilde{\omega}_{c i}^{T}(k+1) \widetilde{\omega}_{c i}(k+1)\right\}-\left(1 / \mathscr{K}_{c i}\right) \operatorname{tr}\left\{\widetilde{\omega}_{c i}^{T}(k) \widetilde{\omega}_{c i}(k)\right\} \\
& =\left(1 / \mathscr{K}_{c i}\right) \operatorname{tr}\left\{\widetilde{\omega}_{c i}^{T}(k+1) \widetilde{\omega}_{c i}(k+1)-\widetilde{\omega}_{c i}^{T}(k) \widetilde{\omega}_{c i}(k)\right\} .
\end{aligned}
$$

According to the update function for the weight matrix

of critic NN (30), we have

$$
\begin{aligned}
\widetilde{\omega}_{c i}(k+1)= & \widetilde{\omega}_{c i}(k)-\mathscr{K}_{c i} \varepsilon_{c i}^{T}(k)\left(-\rho \psi_{c i}\left(\widehat{w}_{c i}^{T} z_{c i}(k+1)\right)+\psi_{c i}\left(\widehat{w}_{c i}^{T} z_{c i}(k)\right)\right) \\
= & \widetilde{\omega}_{c i}(k)-\mathscr{K}_{c i}\left(-\rho \psi_{c i}\left(\widehat{w}_{c i}^{T} z_{c i}(k+1)+\psi_{c i}\left(\widehat{w}_{c i}^{T} z_{c i}(k)\right)\right)\right) \\
& \times\left(-\left(U_{i}(k)+\rho \widehat{\omega}_{c i}^{T} \Psi_{c i}\left(\widehat{w}_{c i}^{T} z_{c i}(k+1)\right)\right)+\widehat{\omega}_{c i}^{T} \Psi_{c i}\left(\widehat{w}_{c i}^{T} z_{c i}(k)\right)\right) \\
= & \widetilde{\omega}_{c i}(k)-\mathscr{K}_{c i} \eta(k)\left(-U_{i}(k)+\widehat{\omega}_{c i}^{T}(\eta(k))\right) \\
= & \left(I-\mathscr{K}_{c i} \eta(k) \eta^{T}(k)\right) \widetilde{\omega}_{c i}(k)+\mathscr{K}_{c i} U_{i}(k) \eta(k),
\end{aligned}
$$

where $\eta(k)=-\rho \Psi_{c i}\left(\widehat{w}_{c i}^{T} z_{c i}(k+1)\right)+\Psi_{c i}\left(\widehat{w}_{c i}^{T} z_{c i}(k)\right)$.

Substituting (38) into 37 we have

$$
\begin{aligned}
\Delta \mathscr{L}_{i, 2}= & \left(1 / \mathscr{K}_{c i}\right) \operatorname{tr}\left\{\begin{array}{c}
\left(\left(I-\mathscr{K}_{c i} \eta(k) \eta^{T}(k)\right) \widetilde{\omega}_{c i}(k)+\mathscr{K}_{c i} U_{i}(k) \eta(k)\right)^{T} \\
\times\left(\left(I-\mathscr{K}_{c i} \eta(k) \eta^{T}(k)\right) \widetilde{\omega}_{c i}(k)+\mathscr{K}_{c i} U_{i}(k) \eta(k)\right)-\widetilde{\omega}_{c i}^{T}(k) \widetilde{\omega}_{c i}(k)
\end{array}\right\} \\
\leq & \left(1 / \mathscr{K}_{c i}\right)\left(\left\|\mathscr{K}_{c i} \eta(k) \eta^{T}(k)\right\|_{F}^{2}\left\|\widetilde{\omega}_{c i}(k)\right\|^{2}+\left\|\mathscr{K}_{c i} U_{i}(k) \eta(k)\right\|^{2}\right) \\
= & \left\|\eta(k) \eta^{T}(k)\right\|_{F}^{2}\left\|\widetilde{\omega}_{c i}(k)\right\|^{2}+U_{i}(k)\|\eta(k)\|^{2} \\
& =\|\eta(k)\|^{4}\left\|\widetilde{\omega}_{c i}(k)\right\|^{2}+U_{i}(k)\|\eta(k)\|^{2} .
\end{aligned}
$$


The difference between $\mathscr{L}_{i, 3}(k+1)$ and $\mathscr{L}_{i, 3}(k)$ can be given as

$$
\begin{aligned}
\Delta \mathscr{L}_{i, 3} & =\left(1 / \mathscr{K}_{a i}^{2}\right) \operatorname{tr}\left\{\widetilde{\omega}_{a i}^{T}(k+1) \widetilde{\omega}_{a i}(k+1)\right\}-\left(1 / \mathscr{K}_{a i}\right) \operatorname{tr}\left\{\widetilde{\omega}_{a i}^{T}(k) \widetilde{\omega}_{a i}(k)\right\} \\
& =\left(1 / \mathscr{K}_{a i}^{2}\right) \operatorname{tr}\left\{\widetilde{\omega}_{a i}^{T}(k+1) \widetilde{\omega}_{a i}(k+1)-\widetilde{\omega}_{a i}^{T}(k) \widetilde{\omega}_{a i}(k)\right\} .
\end{aligned}
$$

According to the update function for the weight matrix of critic NN (34), we have

$$
\begin{aligned}
\widetilde{\omega}_{a i}(k+1) & =\widetilde{\omega}_{a i}(k)-\mathscr{K}_{a i} \varepsilon_{a i}^{T}(k) \widehat{\omega}_{c i} \Omega(k) C_{i} \Psi_{a i}\left(\widehat{w}_{a i}^{T} z_{a i}(k)\right) \\
& =\widetilde{\omega}_{a i}(k)-\mathscr{K}_{a i} \widehat{V}_{i}(k) \widehat{\omega}_{c i} \Omega(k) C_{i} \Psi_{a i}\left(\widehat{w}_{a i}^{T} z_{a i}(k)\right) .
\end{aligned}
$$

Substituting (41) into (40), we have

$$
\begin{aligned}
& \Delta \mathscr{L}_{i, 3}=\frac{1}{\mathscr{K}_{a i}} \operatorname{tr}\left\{\begin{array}{c}
\left(\widetilde{\omega}_{a i}(k)-\mathscr{K}_{a i} \widehat{V}_{i}(k) \widehat{\omega}_{c i} \Omega(k) C_{i} \Psi_{a i}\left(\widehat{w}_{a i}^{T} z_{a i}(k)\right)\right)^{T} \\
\times\left(\widetilde{\omega}_{a i}(k)-\mathscr{K}_{a i} \widehat{V}_{i}(k) \widehat{\omega}_{c i} \Omega(k) C_{i} \Psi_{a i}\left(\widehat{w}_{a i}^{T} z_{a i}(k)\right)\right) \\
-\widetilde{\omega}_{a i}^{T}(k) \widetilde{\omega}_{a i}(k)
\end{array}\right\} \\
& =\frac{1}{\mathscr{K}_{a i}} \operatorname{tr}\left\{\begin{array}{c}
-\widetilde{\omega}_{a i}^{T}(k) \mathscr{K}_{a i} \widehat{V}_{i}(k) \widehat{\omega}_{c i} \Omega(k) C_{i} \Psi_{a i}\left(\widehat{w}_{a i}^{T} z_{a i}(k)\right) \\
-\mathscr{K}_{a i} \widehat{V}_{i}(k) \Psi_{a i}^{T}\left(\widehat{w}_{a i}^{T} z_{a i}(k)\right) C_{i}^{T} \Omega^{T}(k) \widehat{\omega}^{T}{ }_{c i} \widetilde{\omega}_{a i}(k) \\
+\mathscr{K}_{a i}^{2} \widehat{V}_{i}^{2}(k) \Psi_{a i}^{T}\left(\widehat{w}_{a i}^{T} z_{a i}(k)\right) C_{i}^{T} \Omega^{T}(k) \widehat{\omega}^{T}{ }_{c i} \widehat{\omega}_{c i} \Omega(k) C_{i} \Psi_{a i}\left(\widehat{w}_{a i}^{T} z_{a i}(k)\right)
\end{array}\right\} \\
& \operatorname{tr}\left\{\begin{array}{c}
-2 \widehat{V}_{i}(k) \Psi_{a i}^{T}\left(\widehat{w}_{a i}^{T} z_{a i}(k)\right) C_{i}^{T} \Omega^{T}(k) \widehat{\omega}_{c i}^{T} \widetilde{\omega}_{a i}(k) \\
+\mathscr{K}_{a i} \widehat{V}_{i}^{2}(k) \Psi_{a i}^{T}\left(\widehat{w}_{a i}^{T} z_{a i}(k)\right) C_{i}^{T} \Omega^{T}(k) \widehat{\omega}^{T}{ }_{c i} \widehat{\omega}_{c i} \Omega(k) C_{i} \Psi_{a i}\left(\widehat{w}_{a i}^{T} z_{a i}(k)\right)
\end{array}\right\} \\
& =-2 \widehat{V}_{i}(k) \operatorname{tr}\left\{\widetilde{\omega}_{a i}^{T}(k) \widehat{\omega}_{c i} \Omega(k) C_{i} \Psi_{a i}\left(\widehat{w}_{a i}^{T} z_{a i}(k)\right)\right\}+\mathscr{K}_{a i} \widehat{V}_{i}^{2}(k)\left\|\widehat{\omega}_{c i} \Omega(k) C_{i} \Psi_{a i}\left(\widehat{w}_{a i}^{T} z_{a i}(k)\right)\right\|_{F}^{2} .
\end{aligned}
$$

Combining (36), (39), and (42), the difference between $\Delta \mathscr{L}(k)$ and $\Delta \mathscr{L}(k+1)$ is given as

$$
\begin{aligned}
\Delta \mathscr{L} \leq & -\left\|\mathbf{x}_{i}(k)\right\|^{2}-2 \widehat{V}_{i}(k) \operatorname{tr}\left\{\widetilde{\omega}_{a i}^{T}(k) \widehat{\omega}_{c i} \Omega(k) C_{i} \Psi_{a i}\left(\widehat{w}_{a i}^{T} z_{a i}(k)\right)\right\} \\
& +\left\|\mathbf{A}_{i} \mathbf{x}_{i}(k)+\mathbf{B}_{i} \omega_{a i}^{*} \Psi\left(w_{a i} \xi_{i}\right)\right\|^{2}+\|\eta(k)\|^{4}+U_{i}(k)\|\eta(k)\|^{2} \\
& +\mathscr{K}_{a i} \widehat{V}_{i}^{2}(k)\left\|\widehat{\omega}_{c i} \Omega(k) C_{i} \Psi_{a i}\left(\widehat{w}_{a i}^{T} z_{a i}(k)\right)\right\|_{F}^{2} \\
\leq & -\left\|\mathbf{x}_{i}(k)\right\|^{2}-2 \widehat{V}_{i}(k) \operatorname{tr}\left\{\widetilde{\omega}_{a i}^{T}(k) \widehat{\omega}_{c i} \Omega(k) C_{i} \Psi_{a i}\left(\widehat{w}_{a i}^{T} z_{a i}(k)\right)\right\}+D_{m i},
\end{aligned}
$$




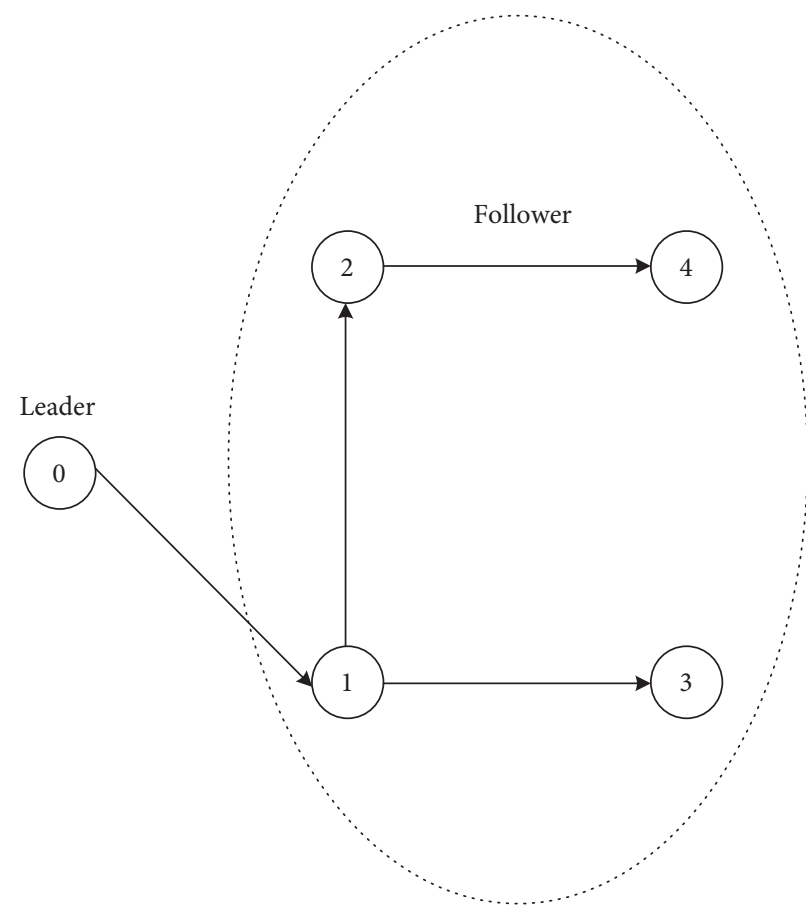

Figure 2: The network topology for the discrete-time MAS.

where $D_{m i}=\sup \left(\left(\left\|A_{i} x_{i}(k)+B_{i} \omega_{a i}^{*} \Psi\left(w_{a i} \xi_{i}\right)\right\|^{2}+\| \eta\right.\right.$ $(k)\left\|^{4}+U_{i} \quad(k)\right\| \eta(k)\left\|^{2}+\mathscr{K}_{a i} \widehat{V}_{i}^{2 i}(k)\right\| \widehat{\omega}_{c i} \Omega\left((k) C_{i} \Psi_{a i}\right.$ $\left.\left.\left(\widehat{w}_{a i}^{T} z_{a i}(k)\right)\right) \|_{F}^{2}\right)$.

If one of the conditions $\|x(k)\| \geq \sqrt{D_{m i}}$ or $\widehat{V}_{i}(k) \operatorname{tr}\left\{\widetilde{\omega}_{a i}^{T}(k) \widehat{\omega}_{c i} \Omega(k) C_{i} \Psi_{a i}\left(\widehat{w}_{a i}^{T} z_{a i}(k)\right)\right\} \geq D_{m i}$ holds, the difference is $\Delta \mathscr{L}<<0$. This means the states of the system and the error of the weight matrices for critic NN and actor NN are UUB.

Case 2: if the ET condition is not satisfied at iteration instant $k$, consider the Lyapunov function (35) in case 1. The difference between $\mathscr{L}_{i, 1}(k+1)$ and $\mathscr{L}_{i, 1}(k)$ can be given as

$$
\begin{aligned}
\Delta \mathscr{L}_{i, 1} & =\mathbf{x}_{i}^{T}(k+1) \mathbf{x}_{i}(k+1)-\mathbf{x}_{i}^{T}(k) \mathbf{x}_{i}(k) \\
& =\left\|\mathbf{x}_{i}(k+1)\right\|^{2}-\left\|\mathbf{x}_{i}(k)\right\|^{2} \\
& \leq-\left\|\mathbf{x}_{i}(k)\right\|^{2}+\left\|L \mathbf{x}(k)+L^{2}\right\| \boldsymbol{\xi}(k)\|\| .
\end{aligned}
$$

The weight matrices for the critic NN and actor NN are not updated when the ET condition is not satisfied, so the differences are $\Delta \mathscr{L}_{i, 2}=0$ and $\Delta \mathscr{L}_{i, 3}=0$.

Combining $\Delta \mathscr{L}_{i, 1}, \Delta \mathscr{L}_{i, 2}$, and $\Delta \mathscr{L}_{i, 3}$, the difference between $\Delta \mathscr{L}(k)$ and $\Delta \mathscr{L}(k+1)$ is given as

$$
\begin{aligned}
\Delta \mathscr{L} & =\Delta \mathscr{L}_{i, 1}+\Delta \mathscr{L}_{i, 2}+\Delta \mathscr{L}_{i, 3} \\
& \leq-\left\|\mathbf{x}_{i}(k)\right\|^{2}+\left\|L \mathbf{x}(k)+L^{2}\right\| \boldsymbol{\xi}(k)\|\| .
\end{aligned}
$$

If the condition $\left\|\mathbf{x}_{i}(k)\right\| \geq \sqrt{\left\|L \mathbf{x}(k)+L^{2}\right\| \xi(k)\|\|}$ holds, the difference is $\Delta \mathscr{L}<0$. This means when the ET condition is not satisfied at the time index $k$, the states of the system and the error of the weights matrices for the critic NN and actor NN are UUB.

\section{Simulation Analysis}

To test the effectiveness of the proposed algorithm, we apply the proposed algorithm in a numerical example. Consider a discrete-time leader-follower MAS consisting of 4 agents with a network topology, as shown in Figure 2. In the topology, agent 0 denotes the leader and the followers are labeled as agent 1 to agent 4 . The adjacency elements $\alpha_{21}, \alpha_{31}$, and $\alpha_{42}$ are set to 1 . The other adjacency elements are set to 0 . In this numerical example, only agent 1 can communicate with the leader, which means $\beta_{1}=1$ and $\beta_{2}=\beta_{3}=\beta_{4}=0$. The weight matrices of the utility function are selected as $Q_{11}=Q_{11}=Q_{11}=Q_{11}=I_{2 \times 2}, \quad R_{11}=R_{22}=R_{33}=R_{44}=1$, and $S_{12}=S_{13}=S_{14}=S_{23}=S_{24}=S_{32}=S_{34}=S_{41}=S_{43}={ }^{0}$.

The dynamics matrix for the leader are set to $\mathbf{A}=\left[\begin{array}{cc}0.9950 & 0.0798 \\ -0.0798 & 0.9950\end{array}\right]$. The dynamics matrices for the $\begin{array}{ll}\text { followers are set to } \mathbf{A}=\left[\begin{array}{cc}0.9950 & 0.0798 \\ -0.0798 & 0.9950\end{array}\right], \\ \mathbf{B}_{1}=\left[\begin{array}{l}0.2047 \\ 0.0898\end{array}\right], \quad \mathbf{B}_{2}=\left[\begin{array}{l}0.2147 \\ 0.2895\end{array}\right], \quad \mathbf{B}_{3}=\left[\begin{array}{l}0.2097 \\ 0.1897\end{array}\right], \quad \text { and } \\ \mathbf{B}_{4}=\left[\begin{array}{l}0.2000 \\ 0.1000\end{array}\right] . & \end{array}$

The parameters for the critic $\mathrm{NN}$ and the actor NN are set to $\rho=0.9$, and $\mathscr{K}_{c 1}=\mathscr{K}_{c 2}=\mathscr{K}_{c 3}=0.01, K_{c 4}=0.001$, and $\mathscr{K}_{a 1}=\mathscr{K}_{a 2}=\mathscr{K}_{a 3}=\mathscr{K}_{a 4}=0.01 .{ }_{-{ }^{2}} \Psi_{c 1}\left(k_{1, R 2}\right)=\left[\tilde{\xi}_{11}^{2}\right.$ $\left.\left(k_{1, p}\right), \widehat{\xi}_{12}^{2}\left(k_{1, p}\right), \widehat{u}_{1}\left(k_{1, p}\right)\right], \Psi_{c 2}\left(k_{2, p}\right)=\left[\hat{\xi}_{21}^{2}\left(k_{2, p p}\right), \xi_{22}\left(k_{2, p}\right)\right.$, $\left.\widehat{u}_{1}^{2}\left(k_{2, p}\right), \widehat{u}^{2}{ }_{2}\left(k_{2, p}\right)\right], \quad \Psi_{c 3}\left(k_{3, p}\right)=\left[\tilde{\xi}_{31_{2}}\left(k_{3, p}\right), \xi_{322}\left(k_{3, p}\right), \widehat{u}^{2}{ }_{1}\right.$ $\left.\left(k_{3, p}\right), \widehat{\vec{u}}_{3}^{2}\left(k_{3, p}\right)\right]$, and $\Psi_{c 4}\left(k_{4, p}\right)=\left[\tilde{\xi}_{41}^{2}\left(k_{4, p}\right), \xi_{42}\left(k_{4, p}\right), \widehat{u}_{2}\right.$ $\left.\left(k_{4, p}\right), \widehat{u}_{4}\left(k_{4, p}\right)\right]$ are the activation functions of the critic NNs. The activation functions of the actor NNs are set to $\Psi_{a i}\left(k_{i, p}\right)=\left[\widehat{\xi}_{i}\left(k_{i, p}\right)\right] . \quad \mathbf{x}_{0}(0)=[0.6311,0.0899]^{T}, \quad \mathbf{x}_{1}(0)$ 


\section{Initialization:}

Give the computation precision $\tau$ and the initial state $x_{i}(0)$ for agent $i$;

Give the initial state $\mathbf{x}_{0}(0)$ for the leader;

Select the learning rate $\mathscr{K}_{a i}$ and $\mathscr{K}_{c i}$;

Give the positive matrices $\mathbf{Q}_{i i}, \mathbf{R}_{i i}$, and $\mathbf{S}_{i j}$;

Initialize the event-triggered error condition $\delta_{i T}(0)=0$;

Select the positive constant $L$;

Iteration:

Let the iteration index $k=0$;

repeat:

Calculate the tracking error $\widehat{\xi}_{i}(k)$ and the event-triggered error $\delta_{i}(k)$;

IF $\left\|\delta_{i}(k)\right\| \geq \delta_{i T}(k)$ :

Event-triggered error $\left\|\delta_{i}(k)\right\|=0$;

Event-triggered index $k_{i, p}=k$;

Compute the control law $\widehat{u}_{i}(k)$;

Compute the local cost function $\widehat{V}_{i}(k)$;

Compute the next state $\hat{x}_{i}(k+1)$ of agent $i$ and the next state $\hat{x}_{0}(k+1)$ of the leader agent;

Calculate the next tracking error $\xi_{i}(k)$;

Compute the control law $\widehat{u}_{i}(k+1)$;

Compute the local cost function $\widehat{V}_{i}(k+1)$;

Update the weights matrix of the critic NN;

Update the weights matrix of the actor NN;

ELSE:

The control law $\widehat{u}_{i}(k)=\widehat{u}_{i}(k-1)$;

Compute the control law $\widehat{u}_{i}(k)$;

Compute the next state $\mathbf{x}_{i}(k+1)$ of agent $i$ and the next sate $\mathbf{x}_{0}(k+1)$ of the leader agent according to the model $\mathrm{NN}$;

$k=k+1$;

End

Until $\left|\widehat{\omega}_{c i}(k+1)-\widehat{\omega}_{c i}(k)\right| \leq \tau$;

Algorithm 1: NN-based event-triggered optimal consensus control algorithm for discrete-time MASs.

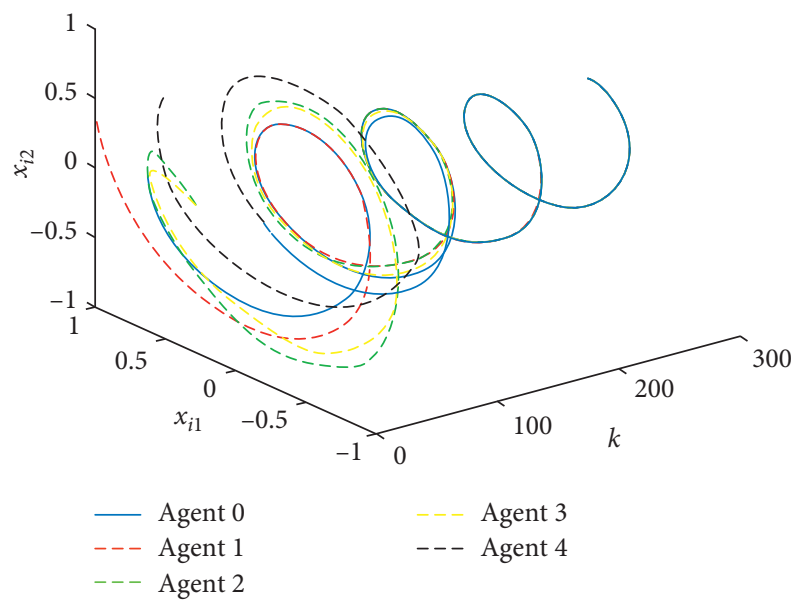

Figure 3: The tracking path for the system under the ET condition.

$=[0.9954,0.3321]^{T}, \quad \mathbf{x}_{2}(0)=[0.2973,0.0620]^{T}, \quad \mathbf{x}_{3}(0)=$ $[0.2982,0.0464]^{T}$, and $\mathbf{x}_{4}(0)=[0.5054,0.7614]^{T}$ are chosen as the initial states for the leader and the follower agents in the system. We set $L=0.1$.

The tracking path for every agent in the discrete-time MAS is shown in Figure 3. From Figure 3, we can observe that all the agents in the system can reach the same state as the leader, and then, they achieve synchronization. The driving errors for the agents in the system are shown in Figure 4. All the agents' driving error all are not updated at every instant $k$, that is to say, all the agents are driven when the ET condition is satisfied. Figure 5 shows the comparisons of event-triggered errors and thresholds for every agent in the system. In Figure 5, we can observe that the eventtriggered errors are always smaller than the thresholds during the tracking process, and we only sample the data 

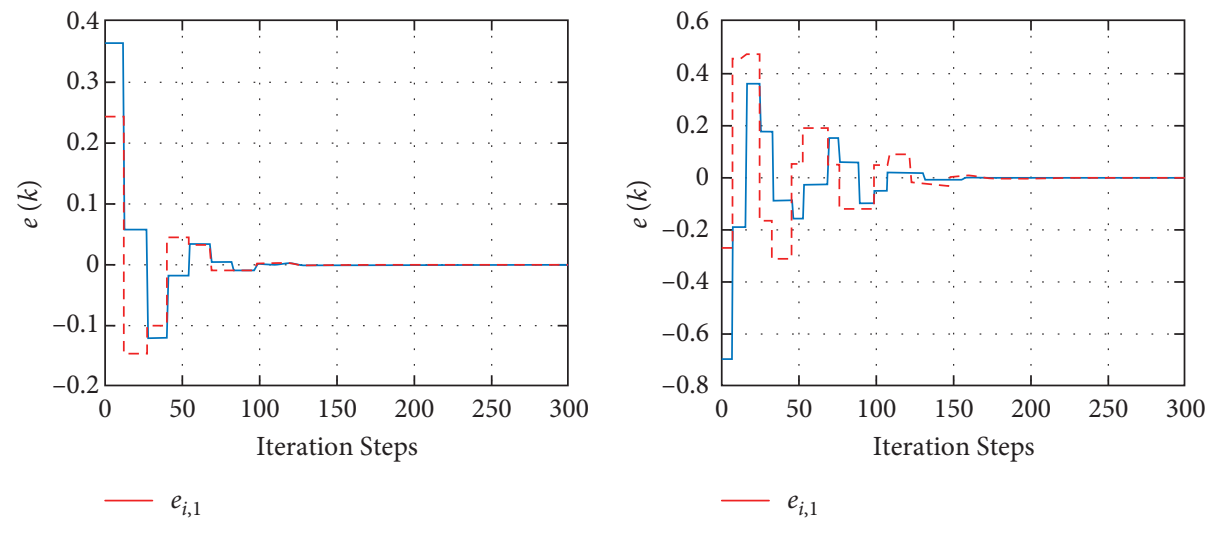

$-e_{i, 2}$

(a)

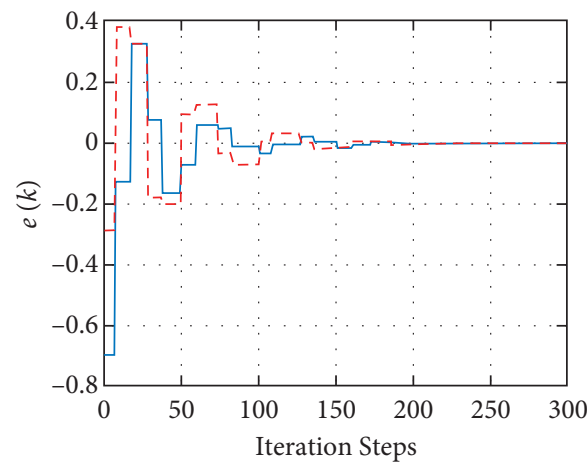

(b)

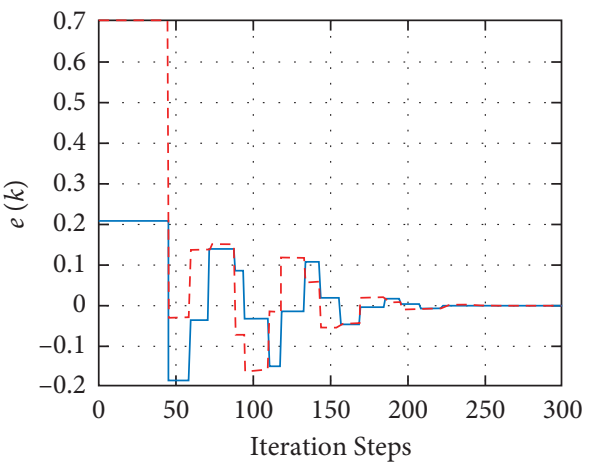

$-e_{i, 1}$

$-e_{i, 1}$

(c)

(d)

FIGURE 4: The driving error for every agent in the system under the ET condition. (a) Driving error for agent1. (b) Driving error for agent2. (c) Driving error for agent3. (d) Driving error for agent4.

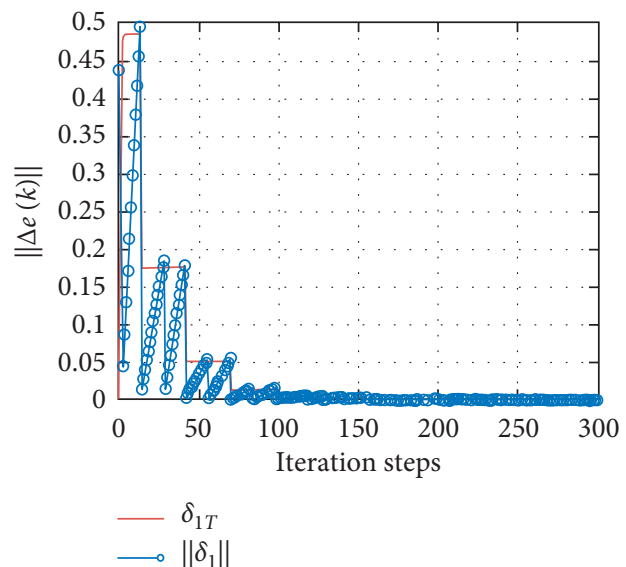

(a)

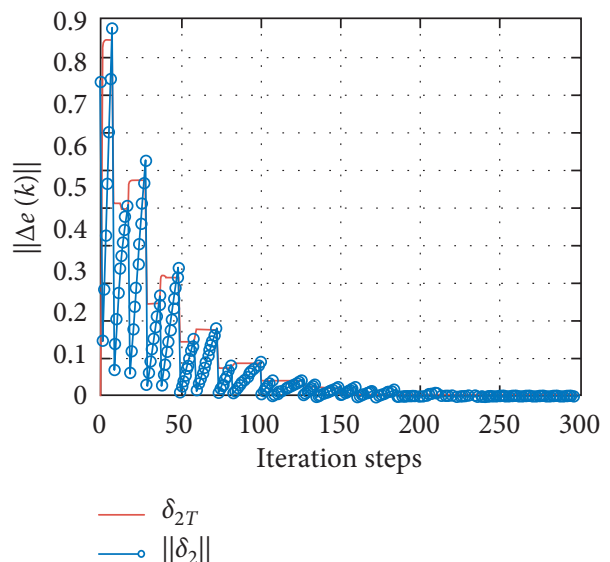

(b)

Figure 5: Continued. 


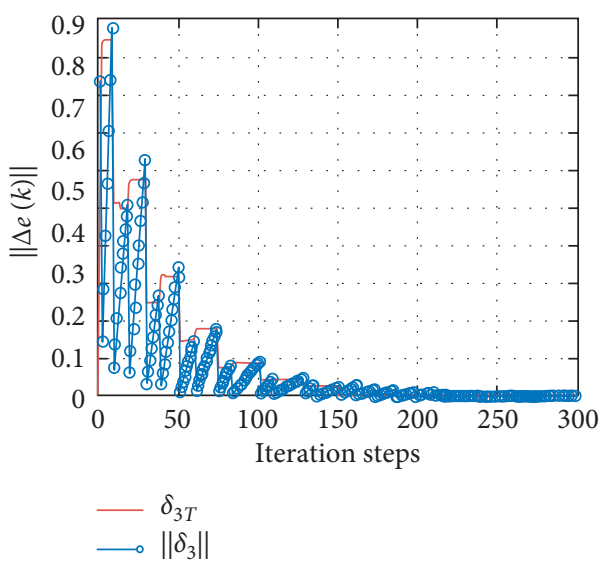

(c)

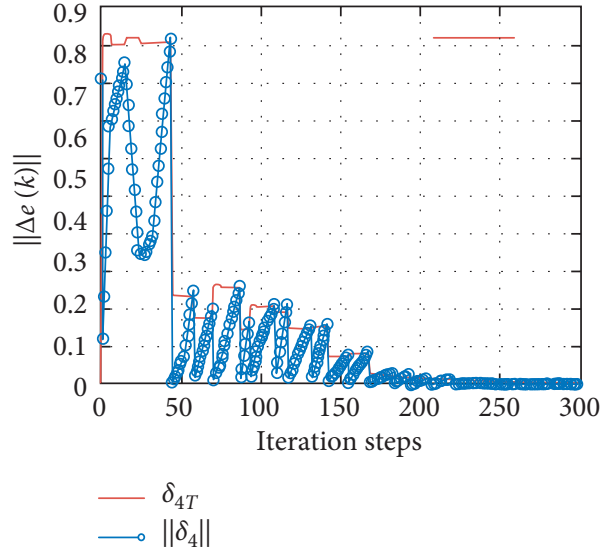

(d)

Figure 5: Comparisons of event-triggered errors and thresholds for every agent in the system. (a) Comparisons of event-triggered errors and thresholds for agent1. (b) Comparisons of event-triggered errors and thresholds for agent2. (c) Comparisons of event-triggered errors and thresholds for agent3. (d) Comparisons of event-triggered errors and thresholds for agent 4.

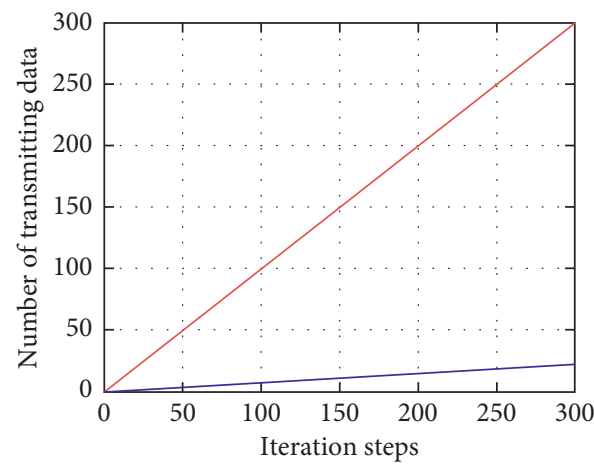

— Time-triggered ADP

— Event-triggered ADP

(a)

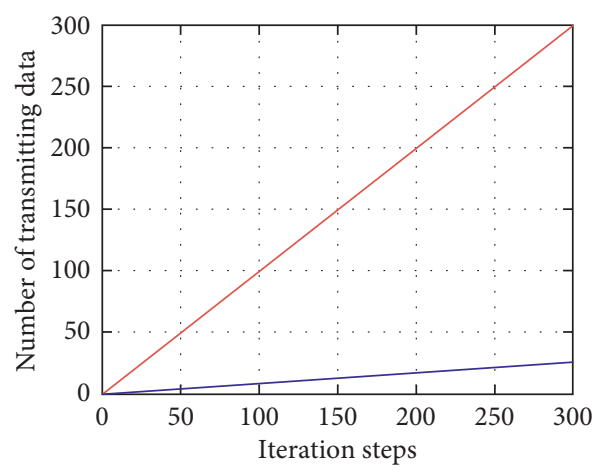

— Time-triggered ADP

— Event-triggered ADP

(c)

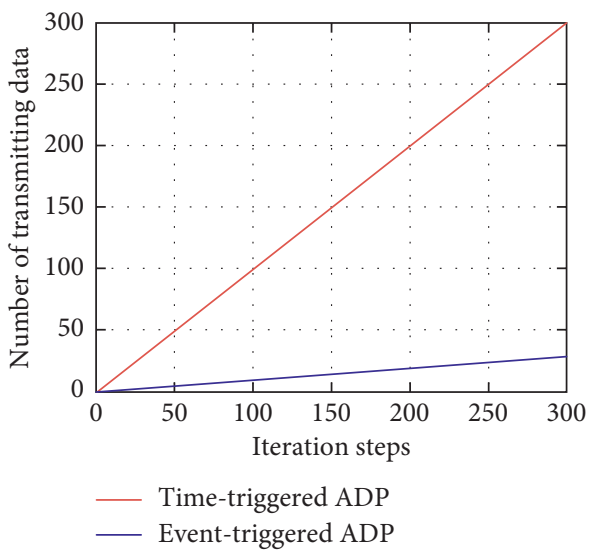

(b)

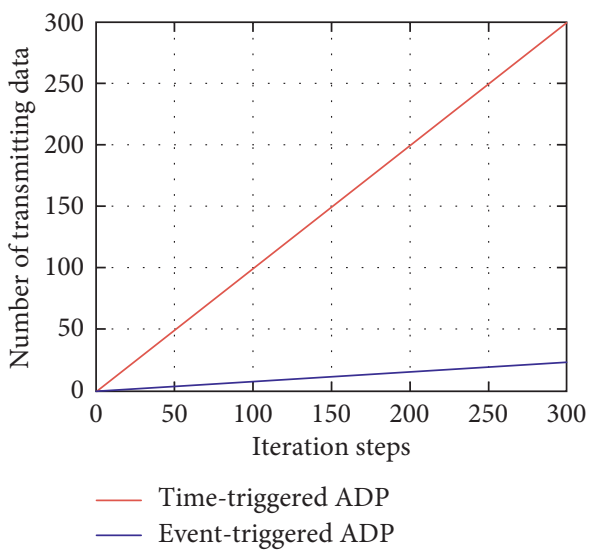

(d)

FIGURE 6: Comparisons of required number of transmitting data under the time-triggered and event-triggered ADP for every agent in the system. (a) The required number of transmitting data under the time-triggered ADP and event-triggered ADP for agent1. (b) The required number of transmitting data under the time-triggered ADP and event-triggered ADP for agent2. (c) The required number of transmitting data under the time-triggered ADP and event-triggered ADP for agent1. (d) The required number of transmitting data under the timetriggered ADP and event-triggered ADP for agent1. 
when the event-triggered errors are bigger or equal to the thresholds, so we sample the less data and save computing resources using our algorithm. Figure 6 shows the comparisons of the required number of transmitting data under the time-triggered and event-triggered ADP algorithm for every agent in the system. We can observe the required number of the event-triggered algorithm is much less than the required number of the time-triggered algorithm.

\section{Conclusion}

An event-triggered optimal consensus tracking control algorithm based on the ADP structure is proposed in this paper. To save the communication and computation resources, we introduce the event-triggered scheme to the optimal consensus tracking control algorithm. The neural networks technology is introduced to simplify the application of the proposed algorithm. It is proved the discretetime MASs are stable with the proposed algorithm and the estimate errors of the weights for NNs are UUB. The simulation results illustrate the efficiency of the proposed method.

\section{Data Availability}

All data included in this study are available from the corresponding author upon request.

\section{Conflicts of Interest}

The authors declare that they have no conflicts of interest.

\section{Acknowledgments}

This work was supported by the National Natural Science Foundation of China (61803285 and 62001332) and National Defense Pre-Research Foundation of China (H04W201018).

\section{References}

[1] Z. Lin, L. Wang, Z. Han, and M. Fu, "Distributed formation control of multi-agent systems using complex laplacian," IEEE Transactions on Automatic Control, vol. 59, no. 7, pp. 17651777, 2014.

[2] Y.-B. Bae, Y.-H. Lim, and H.-S. Ahn, "Distributed robust adaptive gradient controller in distance-based formation control with exogenous disturbance," IEEE Transactions on Automatic Control, vol. 66, no. 6, pp. 2868-2874, 2021.

[3] J. Zhan and X. Li, "Flocking of multi-agent systems via model predictive control based on position-only measurements," IEEE Transactions on Industrial Informatics, vol. 9, no. 1, pp. 377-385, 2013.

[4] C. Yang, G. Peng, L. Cheng, J. Na, and Z. Li, "Force sensorless admittance control for teleoperation of uncertain robot manipulator using neural networks," IEEE Transactions on Systems, Man, and Cybernetics: Systems, vol. 51, no. 5, pp. 3282-3292, 2021.

[5] C. Yang, J. Luo, C. Liu, M. Li, and S.-L. Dai, "Haptics electromyography perception and learning enhanced intelligence for teleoperated robot," IEEE Transactions on Automation Science and Engineering, vol. 16, no. 4, pp. 1512-1521, 2019.
[6] C. Yang, C. Chen, N. Wang, Z. Ju, J. Fu, and M. Wang, "Biologically inspired motion modeling and neural control for robot learning from demonstrations," IEEE Transactions on Cognitive and Developmental Systems, vol. 11, no. 2, pp. 281-291, 2019.

[7] Z. Zuo, J. Zhang, and Y. Wang, "Adaptive fault-tolerant tracking control for linear and lipschitz nonlinear multi-agent systems," IEEE Transactions on Industrial Electronics, vol. 62, no. 6, pp. 3923-3931, 2015.

[8] H. Rezaee and F. Abdollahi, "Adaptive leaderless consensus control of strict-feedback nonlinear multiagent systems with unknown control directions," IEEE Transactions on Systems, Man, and Cybernetics: Systems, vol. 51, no. 10, pp. 6435-6444, 2021.

[9] D. Yao, H. Li, R. Lu, and Y. Shi, "Distributed sliding-mode tracking control of second-order nonlinear multiagent systems: an event-triggered approach," IEEE Transactions on Cybernetics, vol. 50, no. 9, pp. 3892-3902, 2020.

[10] J. Zhang, M. Lyu, T. Shen, L. Liu, and Y. Bo, "Sliding mode control for a class of nonlinear multi-agent system with time delay and uncertainties," IEEE Transactions on Industrial Electronics, vol. 65, no. 1, pp. 865-875, 2018.

[11] D. Liu, H. Li, and D. Wang, "Online synchronous approximate optimal learning algorithm for multi-player non-zerosum games with unknown dynamics," IEEE Transactions on Systems, Man, and Cybernetics: Systems, vol. 44, no. 8, pp. 1015-1027, 2014.

[12] D. Liu and Q. Wei, "Policy iteration adaptive dynamic programming algorithm for discrete-time nonlinear systems," IEEE Transactions on Neural Networks and Learning Systems, vol. 25, no. 3, pp. 621-634, 2014.

[13] B. Mu, K. Zhang, and Y. Shi, "Discrete-time nonlinear HJB solution using approximate dynamic programming," IEEE Transactions on Systems, Man, and Cybernetics, Part B (Cybernetics), vol. 38, no. 4, 2008.

[14] H. Zhang, J. Zhang, G.-H. Yang, and Y. Luo, "Leader-based optimal coordination control for the consensus problem of multiagent differential games via fuzzy adaptive dynamic programming," IEEE Transactions on Fuzzy Systems, vol. 23, no. 1, pp. 152-163, 2015.

[15] H. Zhang, H. Liang, Z. Wang, and T. Feng, "Optimal output regulation for heterogeneous multiagent systems via adaptive dynamic programming," IEEE Transactions on Neural Networks and Learning Systems, vol. 28, no. 1, pp. 18-29, 2017.

[16] W. Gao, Y. Jiang, and M. Davari, "Data-driven cooperative output regulation of multi-agent systems via robust adaptive dynamic programming," IEEE Transactions on Circuits and Systems II: Express Briefs, vol. 66, no. 3, pp. 447-451, 2019.

[17] H. Zhang, H. Jiang, Y. Luo, and G. Xiao, "Data-driven optimal consensus control for discrete-time multi-agent systems with unknown dynamics using reinforcement learning method," IEEE Transactions on Industrial Electronics, vol. 64, no. 5, pp. 4091-4100, 2017.

[18] J. Zhang, H. Zhang, and T. Feng, "Distributed optimal consensus control for nonlinear multiagent system with unknown dynamic," IEEE Transactions on Neural Networks and Learning Systems, vol. 29, no. 8, pp. 3339-3348, 2018.

[19] H. Zhang, D. Yue, C. Dou, W. Zhao, and X. Xie, "Data-driven distributed optimal consensus control for unknown multiagent systems with input-delay," IEEE Transactions on Cybernetics, vol. 49, no. 6, pp. 2095-2105, 2019.

[20] H. Zhang, Y. Liu, G. Xiao, and H. Jiang, "Data-based adaptive dynamic programming for a class of discrete-time systems 
with multiple delays," IEEE Transactions on Systems, Man, and Cybernetics: Systems, vol. 50, no. 2, pp. 432-441, 2020.

[21] R. Song and L. Liu, "Event-triggered constrained robust control for partly-unknown nonlinear systems via ADP," Neurocomputing, vol. 404, no. 3, pp. 294-303, 2020.

[22] S. Xue, B. Luo, D. Liu, and Y. Li, "Adaptive dynamic programming based event-triggered control for unknown continuous-time nonlinear systems with input constraints," Neurocomputing, vol. 396, no. 5, pp. 191-200, 2020.

[23] B. Zhao and D. Liu, "Event-triggered decentralized tracking control of modular reconfigurable robots through adaptive dynamic programming," IEEE Transactions on Industrial Electronics, vol. 67, no. 4, pp. 3054-3064, 2020.

[24] L. Cui, X. Xie, X. Wang, Y. Luo, and J. Liu, "Event-triggered single-network ADP method for constrained optimal tracking control of continuous-time non-linear systems," Applied Mathematics and Computation, vol. 352, pp. 220-234, 2019.

[25] B. Luo, Y. Yang, D. Liu, and H.-N. Wu, "Event-triggered optimal control with performance guarantees using adaptive dynamic programming," IEEE Transactions on Neural Networks and Learning Systems, vol. 31, no. 1, pp. 76-88, 2020.

[26] W. Zhao and H. Zhang, "Distributed optimal coordination control for nonlinear multi-agent systems using event-triggered adaptive dynamic programming method," ISA Transactions, vol. 91, pp. 184-195, 2019.

[27] X. Zhong and H. He, “An event-triggered ADP control approach for continuous-time system with unknown internal states," IEEE Transactions on Cybernetics, vol. 47, no. 3, pp. 683-694, 2017.

[28] J. Sun and T. Long, "Event-triggered distributed zero-sum differential game for nonlinear multi-agent systems using adaptive dynamic programming," ISA Transactions, vol. 110, pp. 39-52, 2021.

[29] S. Xue, B. Luo, and D. Liu, "Event-triggered adaptive dynamic programming for unmatched uncertain nonlinear continuous-time systems," IEEE Transactions on Neural Networks and Learning Systems, vol. 32, no. 7, pp. 2939-2951, 2021.

[30] M. Chen, H. Yan, M. Wang, and Z. Li, "Dynamic eventtriggered consensus for discrete-time multi-agent systems," in Proceedings of the IECON 2021 - 47th Annual Conference of the IEEE Industrial Electronics Society, Toronto, ON, Canada, October 2021.

[31] D. Zhao, T. Dong, and W. Hu, "Event-triggered consensus of discrete time second-order multi-agent network," International Journal of Control, Automation and Systems, vol. 16, no. 1, pp. 87-96, 2009.

[32] A. Eqtami, D. V. Dimarogonas, and K. J. Kyriakopoulos, "Event-triggered control for discrete-time systems," in Proceedings of the 2010 American Control Conference, pp. 47194724, Baltimore, MD, USA, July 2010.

[33] C. Yang, C. Chen, W. He, R. Cui, and Z. Li, "Robot learning system based on adaptive neural control and dynamic movement primitives," IEEE Transactions on Neural Networks and Learning Systems, vol. 30, no. 3, pp. 777-787, 2019.

[34] H. Huang, T. Zhang, C. Yang, and C. L. P. Chen, "Motor learning and generalization using broad learning adaptive neural control," IEEE Transactions on Industrial Electronics, vol. 67, no. 10, pp. 8608-8617, 2020. 